\title{
A New Authorization Model for Workflow Management System Using the RPI-RBAC Model *
}

\author{
Seung Yong Lee ${ }^{1}$, YongMin $\mathrm{Kim}^{2}$, BongNam $\mathrm{Noh}^{2}$, and HyungHyo Lee ${ }^{3 * *}$ \\ ${ }^{1}$ Dept. of Information Security, Chonnam National University, Gwangju, Korea 500-757 \\ bircheathena. chonnam.ac.kr \\ ${ }^{2}$ Dept. of Computer Science, Chonnam National University, Gwangju, Korea 500-757 \\ \{ymkim, bongnam\} @chonnam.ac.kr \\ ${ }^{3}$ Div. of Information and EC, Wonkwang University, Iksan, Korea 570-749 \\ hlee@wonkwang.ac.kr
}

\begin{abstract}
The traditional Role Based Access Control (RBAC) model can be applied to WorkFlow Management System (WFMS) well, but there are some issues. Since the senior roles inherit all the permissions of the junior roles and all the permissions are accumulated for the top senior role, applying the traditional RBAC to WFMS does not meet the access control requirements: least privilege principle, Separation of Duty (SoD). To tackle these, we propose applying Restricted Permission Inheritance RBAC to WFMS authorization and evaluate the advantages and benefits of them in design time and runtime.
\end{abstract}

\section{Introduction}

WorkFlow Management System (WFMS) requires various policies involving access control since it defines the business processes and supports the enforcement of process control over processes. In this paper, when traditional Role Based Access Control (RBAC) is applied to WFMS to enforce an authorization [1], we investigate the problems that occur from inheritance of all permissions to senior roles. Then we evaluate the usefulness and benefits of employing the RPI-RBAC for the enforcement of access control polices in WFMS.

\section{Related Work}

YongHoon et al. introduced the concept of the sub-role to restrict the complete inheritance of junior permissions to senior roles [2]. In RBAC models, a senior role inherits the permissions of all its junior roles. One of the main security principles of RBAC models is to enable compliance with the least privilege principle. This unconditional permission inheritance can cause the violation of this principle. In order

\footnotetext{
* This paper was supported by University IT Research Center Project and partially by Wonkwang University in 2001

*** Correspondent author
}

M. Bubak et al. (Eds.): ICCS 2004, LNCS 3036, pp. 639-643, 2004.

(C) Springer-Verlag Berlin Heidelberg 2004 
to address this drawback, they divide a role into a number of sub-roles based upon the characteristics of job functions and the degree of inheritance.

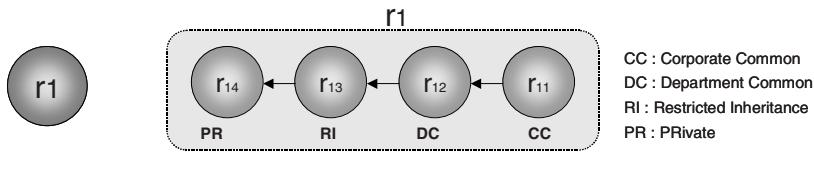

(a) role

(b) sub-roles

Fig. 1. Sub-role concept for corporate environment

Fig. 1. describes their proposed sub-role concept compared with the original role one in the traditional RBAC model.

\section{Applying the RPI-RBAC Model to WFMS}

In this section we mention a workflow scenario in a bank as an example the need for permission restrictions. We will use this scenario to illustrate how the RPI-RBAC model can be applied to this business process well.

Assume that a customer applies for a large loan from a bank. The deposit department uses the customer's credit information which it received from the credit review department to determine the amount of the money to loan to the customer, and a deposit clerk gives the money to the customer while a senior employee checks that the transfer was made successfully.

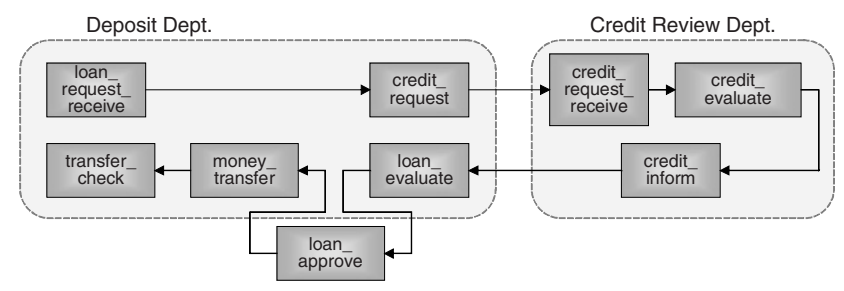

Fig. 2. Business process of loan in a bank

Fig. 2. shows the flow of tasks in the business process related to our scenario. There are two departments: the deposit and credit review departments in the bank scenario. Some tasks like loan_request_receive, credit_request and transfer_money are processed by the members of the deposit department whereas some tasks like credit_request_receive and credit_evaluae are executed by the members of the credit department. These business rules ensure that the Separation of Duty (SoD) requirement is met and that semantic integrity is maintained. In the rules, there are several constraints like who should do something, who can do something, who should not, etc.

In the case of employing traditional RBAC to WFMS, in which the senior roles inherit all the permissions of their junior roles, since the general manager has all the 
permissions of a manager, a supervisor and each departmental clerk, he or she has the permissions needed to execute all the tasks of all junior roles. This runs counter to the principle of least privilege and can cause many problems like abuse of rights and fraud. So, there is a need to restrict permission inheritance to satisfy the least privilege principle and prohibit an overuse of rights. Since the RPI-RBAC model limits the inheritance of permissions from a junior's rights, we can forbid the individual from committing frauds and improperly using his or her rights by applying RPI-RBAC to WFMS authorization.

We can consider the task's functionality, responsibility and interoperability to categorize them. These tasks can be categorized to four categories: Private User Task, Department Task, Restricted Task and User Task. We can assign each task to the proper sub-roles related to the jobs or tasks in design time. Because the permissions assigned to private user tasks should not be inherited by senior roles, if private user tasks are assigned to the private sub-role, we can prevent senior roles from executing the junior's own tasks. Fig. 3. shows the assignment of tasks to sub-roles.

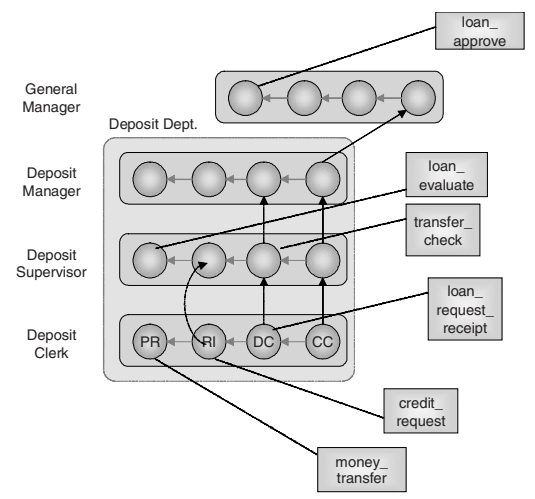

Fig. 3. Detailed assignment of tasks to sub-role

In this part, we examine the application of RPI-RBAC to WFMS authorization from the perspective of runtime and SoD. If it is possible that SoD will be violated by a task in a business process, WFMS prevents the task from being performed or voids the workflow instance by checking the associated constraints.

Assume that there is a business rules that no single individual should be allowed to process two tasks: money_transfer and transfer_check. If someone who executed a money_transfer can also execute a transfer_check, it is possible that he or she could commit fraud. This does not satisfy the requirement of SoD in the business processes.

In the case of applying traditional RBAC to WFMS authorization, the supervisor and other senior users have permission to execute both of the conflicting tasks. To satisfy the business rules described for the integrity, it is necessary to prevent a single user from executing both tasks. To cope with this requirement in traditional RBAC, some type of constraints must be defined and enforced during runtime. Bertino, Ferrari and Atluri (BFA) have introduced authorization constraints into WFMS authorization to deter fraud [3]. Sandhu et al. also presented a Transaction Control 
Expression (TCE) for dynamic SoD [4]. In Table 1, we can use both BFA and TCE to resolve a SoD requirement. These authorization constraints require that any single user should not execute both money_transfer and transfer_check tasks.

Table 1. Constraints to tasks for SoD with BFA and TCE

Constraint by BFA
cannot_do $_{\mathrm{u}}\left(\right.$ transfer_check, $\left.\mathrm{U}_{\mathrm{i}}\right) \leftarrow \operatorname{execute}_{\mathrm{u}}\left(\mathrm{U}_{\mathrm{i}}\right.$, money_transfer, $\left.\mathrm{k}\right)$
Constraint by TCE
money_transfer $\bullet$ deposit_clerk
transfer_check $\bullet$ deposit_supervisor

From the perspective of WFMS authorization, applying the traditional RBAC model causes extra overhead on WFMS, since at runtime the WFMS must monitor the tasks and check the predefined constraints for the integrity during workflow execution time. This inefficiency is caused by the automatic permission inheritance in traditional RBAC and the measures that must be taken to circumvent it. If the RPIRBAC model is applied to WFMS authorization, the administrator does not need to set or configure constraints for the integrity and WFMS do not need to check them for authorization since the RPI-RBAC model itself covers the authorization problems associated with these permissions. So, applying RPI-RBAC to WFMS is more efficient than applying traditional RBAC. However, the former is not as flexible as the later because it strictly limits the inheritance of permissions.

\section{Conclusions}

We have shown that RPI-RBAC satisfies the least privilege principle and separation of duty which are requirement needed for access control in WFMS, and that it works well when applied to WFMS authorization as an access control policy.

If traditional RBAC is applied to WFMS, some integrity constraints to WFMS must be defined and checked during workflow runtime to guarantee the integrity of business processes. But if RPI-RBAC is applied to WFMS, each role has the least amount of permissions and it does not have permission to other private ones. So, it can cut down on the possibility of rights abuse and fraud in advance and thus reduce the overhead of performing the constraints checks in runtime and satisfy SoD.

\section{References}

1. David F. Ferraiolo, D. Richard Kuhn and Ramaswamy Chandramouli, "Role-Based Access Control," Artech House Publishers, ISBN 1-58053-370-1 
2. YongHoon Yi, Myongjae Kim, etc., "Applying RBAC Providing Restricted Permission Inheritance to a Corporate Web Environment," APWeb Conference, Lecture Notes in Computer Science(LNCS) 2642, Sep. 2003, pp. 287-292.

3. Elisa Bertino, Elena Ferrari and Vijay Atluri, "The Specification and Enforcement of Authorization Constraints in Workflow Management Systems," ACM Transactions on Information and System Security, Vol. 2, No. 1, Feb. 1999, pp. 65-104.

4. Savith Kandala, Ravi Sandhu, "Extending the BFA Workflow Authorization Model to Express Weighted Voting,” Database Security XIII: Status and Prospects, Kluwer 2000. 